\title{
Prevalencia de coccidios en pollos de traspatio de Salamanca (Guanajuato, México)
}

\author{
Ramos, D.F.; Sahagún, C.A.; Avila, R.A. \\ División de Ciencias de la Vida, Departamento de Veterinaria y Zootecnia, Universidad \\ de Guanajuato, Campus Irapuato-Salamanca, México. E-mail: ledifar@hotmail.com
}

\begin{abstract}
Resumen
Ramos, D.F.; Sahagún, C.A.; Avila, R.A.: Prevalencia de coccidios en pollos de traspatio de Salamanca (Guanajuato, México). Rev. Vet. 30: 1, 59-62, 2019. El objetivo de la investigación fue determinar la prevalencia de coccidios en pollos de un municipio mexicano. Se tomaron muestras de 97 aves en 17 localidades y se recabaron datos para conocer la cantidad de aves por unidad, el tipo de alimentación, la causa de muerte, la época de mayor mortalidad y la realización de controles antiparasitarios en las 40 unidades visitadas. Se confirmó la presencia de ooquistes de Eimeria sp en 65 muestras, con prevalencia del 67,01\% y niveles de infestación bajos, medios y altos (46, 10 y 9 casos). Hubo correlación entre hembras versus control de parásitos $(p \leq 0,05)$, nivel de infestación versus causa de muerte $(p \leq 0,05)$ y machos versus época de muerte $(\mathrm{p} \leq 0,05)$. El odds ratio indicó que las hembras son más susceptibles que los machos a contraer coccidios $(1,87, \mathrm{p}=0,1883)$. Se concluye que la prevalencia del protozoario en aves de unidades de traspatios en Salamanca es alta y está relacionada con el control parasitario, la época del año y el sexo de las aves. Además, comparadas con los machos, las hembras fueron más susceptibles a contraer coccidios.
\end{abstract}

Palabras clave: pollo, Eimeria sp, prevalencia, control parasitario.

\begin{abstract}
Ramos, D.F.; Sahagún, C.A.; Avila, R.A.: Prevalence of coccidia in backyard chickens of Salamanca (Guanajuato, México). Rev. Vet. 30: 1, 59-62, 2019. The aim of this research was to determine the prevalence of coccidia in chickens from a Mexican municipality. Samples from 97 birds were collected in 17 locations and data regarding number of chickens per location, feeding type, cause of death, seasonal concentration of deaths, and parasitic control of the 40 units visited. The presence of Eimeria sp oocysts was detected in 65 samples with a prevalence of $67.01 \%$, with low, medium and high infestation levels (46, 10 and 9 cases). There was correlation between females versus parasite control $(p \leq 0.05)$, levels of oocysts vs cause of death $(\mathrm{p} \leq 0.05)$, and male vs period of death $(\mathrm{p} \leq 0.05)$. Odds ratio indicates that females were more susceptible than the males to get coccidia $(1.87, \mathrm{p}=0.1883)$. It can be concluded that the prevalence of coccidia in chickens of backyard units in Salamanca is high, and that it is related to the control of parasites, time of the year, and sex of the birds. In addition, females were more susceptible to infestation compared to males.
\end{abstract}

Key words: chicken, Eimeria sp, prevalence, parasite control.

\section{INTRODUCCIÓN}

En las unidades de traspatio se obtienen productos alimenticios para consumo humano y animal. Los excedentes se venden para ayudar al sustento familiar ${ }^{10}$. Las características que las distinguen son carencia de infraestructura y manejo deficiente de los animales. Como resultado, las condiciones de higiene son precarias y las enfermedades proliferan ${ }^{5}$.

La coccidiosis es una enfermedad parasitaria causada por un protozoario cosmopolita de la familia $E i$ meriidae, género Eimeria ${ }^{18}$. Este parásito vive en el

Recibido: 20 noviembre 2018 / Aceptado: 8 febrero 2019 intestino de las aves (un solo huésped), donde desarrolla su ciclo biológico. Las aves se infectan al ingerir ooquistes del suelo, que invaden su intestino como ooquistes, especialmente en las criptas de Lieberkühn. Los signos clínicos dependen de la cantidad de ooquistes ingeridos, así como del estado de salud de las aves, que en los casos severos pueden morir ${ }^{12,16,17}$.

Diferentes estudios se han realizado para establecer la prevalencia de Eimeria $s p$ en aves domésticas de varias regiones del mundo, con resultados variables. Sucesivamente fueron reportadas prevalencias en Chile $(76,5 \%)^{2}$, México $(53 \%){ }^{17}$, Nigeria $(33,3 \%)^{7}$ y Venezuela $(17 \%)^{4}$. Los investigadores indicaron que los 
resultados varían de acuerdo a la temperatura ambiente, humedad relativa y lugar de estudio.

México es un país privilegiado debido a su biodiversidad, la cual se debe a su posición biogeográfica, tipo de territorio y variedad climática ${ }^{15,17}$. Tales características son ideales para desarrollar e incrementar la diversidad biológica, aunque también posibilita la aparición de enfermedades. El suelo, la humedad y la temperatura ambiente son factores que pueden aumentar el desarrollo de parásitos.

El objetivo de la investigación fue determinar la prevalencia de Eimeria $s p$ en aves de traspatio en el Municipio de Salamanca (Guanajuato, México).

\section{MATERIAL Y MÉTODOS}

Área de estudio. La investigación se realizó en el municipio de Salamanca (Guanajuato, México), el cual posee una extensión territorial de $744 \mathrm{~km}^{2}$ distribuida en 256 localidades, ubicado en $20^{\circ} 34^{\prime} 13^{\prime \prime} \mathrm{N}$, $101^{\circ} 11^{\prime} 50^{\prime \prime} \mathrm{W}$, a $1,721 \mathrm{msnm}$. La temperatura promedio anual de la región es de $19^{\circ} \mathrm{C}$ con precipitación pluvial anual de $750 \mathrm{~mm}$.

Tamaño de la muestra. El tamaño de la muestra se determinó con el método de poblaciones infinitas ${ }^{1}$. Fórmula: $\mathrm{N}=\mathrm{Z}^{2} \times \mathrm{p} \mathrm{x} \mathrm{q} / \mathrm{d}^{2}$. Donde: $\mathrm{N}=$ tamaño de la muestra, $Z=$ nivel de confianza $(Z=0.5=1.96), p=$ probabilidad $=0.5, \mathrm{q}=(1-\mathrm{p}) \mathrm{y} \mathrm{d}=$ nivel de presión: $10 \%$ $=0.1$. Entonces $\mathrm{N}=(1.96)^{2} \times 0.5 \times 0.5 /(0.1)^{2}=97$ aves.

Muestras biológicas. Para colectar las heces, las aves se colocaron en una jaula de $50 \times 60 \mathrm{~cm}$ con piso de malla. En la parte inferior se colocó una bolsa plástica. Cuando las aves defecaron, las excretas se colectaron para almacenarse con formol al 10\% (relación 1:1), rotuladas con la fecha, la localidad de procedencia y el sexo del animal, conservándose en refrigeración hasta su análisis ${ }^{6}$. Todos los procedimientos descritos en la investigación respetan los lineamientos establecidos para el manejo de los animales.

Recolección de la información. Durante la visita a la unidad productiva se colectó información entrevistando al encargado para conocer el número de aves, su alimentación; destino comercial, casero o su combinación, causa común de muerte de las aves; enfermedad o descuido, época del año con mayor mortalidad (exceso de calor, lluvia o frío) y finalmente se indagó acerca de la realización de controles antiparasitarios.

Diagnóstico de ooquistes de Eimeria sp. Para determinar la presencia de ooquistes de Eimeria $s p$ en heces se uso la técnica de flotación por centrifugación ${ }^{3,18}$. Dos gramos de heces se colocaron en una solución salina sobresaturada, la cual fue centrifugada durante seis minutos a $1.000 \mathrm{rpm}$. Luego, dos gotas de la parte superior de la solución obtenida se colocaron sobre un por- taobjetos, observándose la preparación a 100 aumentos para identificar presencia o ausencia de ooquistes.

Técnica de McMaster. A las muestras positivas a Eimeria $s p$ se les determinó la cantidad de ooquistes por gramo con la técnica de McMaster ${ }^{3}$. Dos gramos de heces se colocaron en $28 \mathrm{ml}$ de solución salina saturada, mezclándose y luego filtrándose través de un colador plástico. Con una muestra $(1 \mathrm{ml})$ de la parte superior de la solución se llenaron los compartimientos de la cámara de McMaster. La muestra se dejó reposar durante 5 minutos, examinándose a 100 aumentos para contar los ooquistes presentes en cada carril y determinar la cantidad de ooquistes totales ${ }^{3,20}$.

Análisis estadístico. Se determinó la prevalencia de individuos positivos de la población estudiada, en el periodo de tiempo (un año). La información obtenida en las encuestas se correlacionó usando una escala nominal, considerando el nivel de significancia de $p<0,05$. El odds ratio se obtuvo por el método convencional.

\section{RESULTADOS Y DISCUSIÓN}

Presencia de ooquistes de Eimeria sp. En la Tabla 1 se presentan, para cada nivel de infestación (hasta $10,11-20$ y 21 o más ooquistes) ${ }^{7}$, la cantidad de aves portadoras de ooquistes de Eimeria $s p$ y la prevalencia (\%) en machos y hembras. La prevalencia total fue de $67.01 \%$ (machos $25.77 \%$ y hembras $41.24 \%$ ). La elevada presencia de coccidios implica posibilidad de pérdidas económicas debido a la baja productividad de los animales y en casos severos, su muerte.

Los resultados de la investigación indicaron que en Guanajuato la presencia de coccidios fue mayor que la registrada en otras regiones del país, que acusaron niveles de 17 y $53 \%{ }^{4,17}$. En unidades comerciales se reportaron mayores incidencias $\left(75,6\right.$ y 92,3\%) ${ }^{2,11}$. La mayor prevalencia en ejemplares de producción intensiva quizás se deba a la mayor cantidad de aves y su manejo, dadas las condiciones diferentes a las presentadas en aves de traspatio.

En la región, el desarrollo de enfermedades parasitarias puede ocurrir por la época del año, debido a la modificación de las condiciones ambientales; generalmente se reportan prevalencias bajas en épocas de estiaje (sequía) y altas en épocas de lluvia ${ }^{4,8}$.

La alta humedad del ambiente (90\%) prolonga la subsistencia de los ooquistes (52 días), provocando mayor reinfección de las aves. Con menor humedad $(60 \%)$ la vida de los ooquistes es menor a 30 días ${ }^{13}$. En la presente experiencia, la humedad registró alta incidencia durante los muestreos, incrementando la prevalencia de ooquistes en las aves.

Eimeria sp vs unidades de traspatio. En la Tabla 2 se muestra la correlación entre aves positivas, niveles de infestación en hembras y machos con cantidad de aves en la unidad, tipo de alimentación, causa de 
Tabla 1. Nivel de infestación, aves positivas a ooquistes de Eimeria $s p$ y prevalencia en machos y hembras.

\begin{tabular}{ccccccc}
\hline nivel de & \multicolumn{3}{c}{ positivos } & \multicolumn{3}{c}{ prevalencia (\%) } \\
\cline { 2 - 7 } infestación & machos & hembras & total & machos & hembras & total \\
\hline A & 20 & 26 & 46 & 20,62 & 26,80 & 47,42 \\
B & 2 & 8 & 10 & 2,06 & 8,25 & 10,31 \\
C & 3 & 6 & 9 & 3,09 & 6,19 & 9,28 \\
total & 25 & 40 & 65 & 25,77 & 41,24 & 67,01 \\
\hline
\end{tabular}

$\mathrm{A}=1$ a 10 ooquistes, $\mathrm{B}=11$ a 20 ooquistes, $\mathrm{C}=21$ o más ooquistes ${ }^{7}$.

Tabla 2. Correlación entre hembras y machos con cantidad de aves, tipo de alimentación, ocurrencia y época de muerte y control de parásitos.

\begin{tabular}{lccccc}
\hline niveles & cantidad & alimentación & muerte & época & control \\
\hline hembras & $-0,23148$ & 0,01906 & 0,02552 & $-0,05594$ & 0,32417 \\
nivel I & $-0,22621$ & $-0,10564$ & $-0,01375$ & $-0,10616$ & 0,0441 \\
nivel II & $-0,29395$ & $-0,06757$ & 0,35377 & $-0,02587$ & $-0,15323$ \\
& & & 0,0271 & & \\
nivel III & 0,00641 & 0,03704 & 0,3296 & 0,02256 & 0,08792 \\
machos & $-0,07583$ & $-0,00664$ & 0,01936 & 0,33454 & 0,07767 \\
nivel I & $-0,07283$ & $-0,0202$ & $-0,19147$ & 0,22503 & 0,18428 \\
nivel II & $-0,00735$ & 0,00772 & $-0,11658$ & 0,17589 & 0,16129 \\
& & & & & \\
nivel III & 0,02227 & 0,05266 & 0,15534 & $-0,02352$ & $-0,09165$ \\
\hline
\end{tabular}

Cifras cursivas indican correlación significativa $(\mathrm{p} \leq 0,05)$. Nivel I: aves con 1 a 10 ooquistes, Nivel II: con 11 a 20 ooquistes y Nivel III: más de 20 ooquistes.

Tabla 3. Odds ratio de prevalencia de Eimeria $s p$ en hembras y machos en unidades de traspatio.

\begin{tabular}{lccc}
\hline & casos & controles & IC \\
\hline hembra & $40(61,54 \%)$ & $24(75 \%)$ & $0,2076-1,3699$ \\
macho & $25(38,46 \%)$ & $8(25 \%)$ & $0,1795-1,4799$ \\
total & 65 & 32 & \\
\hline
\end{tabular}

IC $=$ intervalo de confianza del $95 \%$

muerte, época de muerte y control de parásitos. Hubo correlación $(p<0,05)$ entre hembras positivas y control de parásitos, mortalidad en hembras con nivel de infestación II y III $(\mathrm{p}<0,05)$ y -en machos- correlación $(p<0,05)$ con la época de su muerte.

Los datos categóricos se utilizan para medir una variable a una escala determinada y permiten clasificar la información en un número limitado de grupos. En la presente investigación se compararon los niveles de infestación con las características de la unidad productiva. Los resultados indicaron mayores prevalencias de coccidiosis en las unidades de producción que no controlaban las parasitosis.

Realizar periódicamente el control de parásitos puede ayudar a disminuir la prevalencia de la enfer- medad ${ }^{4}$. Las aves infectadas eliminan ooquistes continuamente y los ingieren al momento de tomar sus alimentos del suelo contaminado ${ }^{9}$.

La prevalencia de coccidios se ha relacionado con el sexo de sus hospederos, considerando a las hembras más susceptibles ${ }^{9}$ . Además, la humedad favorece la esporulación de los ooquistes, por lo cual en época de lluvias hay mayor infestación de hembras y machos, explicando los resultados de la presente investigación.

Odds ratio. En la Tabla 3 se presentan los odds ratio de Eimeria $s p$ en hembras y machos de traspatio. Surge que las hembras poseen más probabilidad de albergar coccidios $(1,87)$ comparada con los machos (chi cuadrado $=1,7311$, probabilidad $=0,1883$, $\mathrm{n}=97$ ).

Odds ratio es una medida de asociación expresada en términos de posibilidad de ocurrencia de un evento en individuos que cuentan con el factor de riesgo, comparado con aquellos que no lo presentan. En las hembras, el sexo está relacionado con la mortalidad causada por diferentes enfermedades. Los resultados aquí obtenidos indican que las hembras poseen mayor probabilidad de contraer coccidios, comparada con los machos. Resultados similares han sido reportados en diferentes investigaciones 9,14 . El nivel de infestación en nuestro estudio se puede atribuir al clima de la zona y a la época del año en que se realizó el estudio ${ }^{7,9,19}$.

Se concluye que la prevalencia de coccidios en pollos de traspatio de Salamanca (Guanajuato, México) es alta $(67,01 \%)$ y que las hembras son más susceptibles a contraer la enfermedad. Por otra parte, mayor cantidad de machos muere en época de lluvias.

\section{REFERENCIAS}

1. Aguilar BS. 2005. Fórmulas para el cálculo de la muestra en investigaciones de salud. Salud en Tabasco 11: 333-338.

2. Alcaíno H, González JP, Fredes F, Gorman T. 2002. Coccidias aviares de gallineros industriales de Chile. $\mathrm{Pa}$ rasitol Latinoam 57: 34-39.

3. Besné MA, Figueroa CJ, Quiroz RH, Ramírez GA, Ramos ME. 2011. Manual de prácticas de laboratorio de parasitología. Ed. Departamento de Parasitología de la UNAM, México, p. 39-45.

4. Brown E, Díaz CD, Moreno L, Gotopo A. 2006. Prevalencia de Eimeria $s p$ en gallinas ponedoras de granjas pertenecientes a tres municipios del estado Trujillo, Venezuela. Rev Cient 16: 579-584.

5. Caicedo RE, Garita JL, Paz NM. 2011. Salud animal de una cuenca lechera bajo el sistema de traspatio, Puebla, México. Acta Iberoamer Conserv Anim (AICA) 1: 323-326. 
6. Coffin DL. 1959. Laboratorio Clínico en Medicina Veterinaria, 3ra. ed., Cornell University, Ithaca (New York), p. 23-24.

7. Jatau ID et al. 2014. Prevalence of coccidia infection and preponderance Eimeria species in free range indigenous and intensively managed exotic chickens during hot-weet season in Zaria, Nigeria. Asian J Poult Sci 6: 79-88.

8. Kaboundi K, Umar S, Tanveer MM. 2016. Prevalence of coccidiosis in free-range chiken in Sidi Thabet, Tunisa. Scientifica $1-6$.

9. Lawal JR et al. 2016. Dry season prevalence of avian coccidia infection in domesticated chickens (Gallus domesticus) in Jere Council, Borno state, Nigeria. J Anim Sci Vet Med 1: 67-73.

10. López JL, Damián MA, Álvarez F, Parra F, Zuluaga GP. 2018. La economía de traspatio como estrategia de supervivencia en San Nicolás de los Ranchos, Puebla, México. Revista de Geografía Agrícola 48: 49-63.

11. Martínes NS, Bohórquez N. 1994. Prevalence and associate factors to cocidiosis in broilers. Rev Cientifica 4: 25-36.

12. Merk KC, Kahn M. 2007. Manual Merck de Veterinaria, 6ta ed., Océano, Madrid, p. 2169-2171.

13. Moreno DR, Ibarra VF. 2002. Algunos aspectos de la coccidiosis aviar en la zona de Coatzacualcos, Veracruz, México. Vet Méx 33: 63-71.
14. Oljira D, Melaku A, Bogale B. 2012. Prevalence and risk factors of coccidiosis in poultry farms in and around Ambo Town, Western Ethiopia. Am-Euras J Sci Res 7: 146-149.

15. Ramamoorthy TP, Bye R, Lot AJ. 1998. Diversidad biológica de México: orígenes y distribución, Ed. Instituto de Biología, Universidad Nacional Autónoma de México, p. 792.

16. Rodríguez VR, Cob LA, Domínguez JL. 2001. Frecuencia de parásitos gastrointestinales en animales domésticos diagnosticados en Yucatán, México. Rev Biomed 12: 19-25.

17. Sarukhán J et al. 2009. Capital natural de México: conocimiento actual, evaluación y perspectivas de sustentabilidad. Ed. Comisión Nacional para el Conocimiento y Uso de la Biodiversidad, México, p.100.

18. Shapiro LS. 2010. Pathology and parasitology for veterinary technicians, $2^{\text {nd }}$ edition, Delmar (USA), p. 215-228.

19. Yousuf M, Tak H, Gul N. 2013. Prevalence of eimeriosis in domestic/free range chickens of South Kashmir, India. Int J Curr Res 5: 1606-1608.

20. Zajac A, Conboy G. 2012. Veterinary Clinical Parasitology, $8^{\text {th }}$ ed., Wiley-Blackwel (USA), p. 9-11.

\section{Asociación Cooperadora de la Facultad de Ciencias Veterinarias Universidad Nacional del Nordeste}

Personería Jurídica No 647/92 y 912/00

Sargento Cabral 2139

3400 Corrientes

Argentina

La Asociación Cooperadora de la Facultad de Ciencias Veterinarias de la UNNE fue constituida el 10 de diciembre del año 1991 como entidad de bien público, con el objeto de promover y coadyuvar las actividades científicas, educativas y culturales relacionadas con las Ciencias Veterinarias. En tal sentido, implementa acciones para colaborar con la enseñanza, extensión, actualización y difusión científica que realiza dicha casa de estudios.

\section{Beneficios que brinda a sus asociados:}

- Fotocopias con descuentos especiales en la Fotocopiadora Copias.com que funciona dentro del predio de la Facultad de Ciencias Veterinarias.

- Descuentos para la adquisición de libros de distintas editoriales.

- Descuentos especiales en otros rubros.

Tel. (0379) 4425753 interno 186 • Página Web www.vet.unne.edu.ar•E-mail: cooperadora@vet.unne.edu.ar 